\title{
Pharmaceutica Analytica Acta
}

\section{Drug Analysis in Body Fluids}

\section{Hideharu Shintani ${ }^{\star}$}

In the presale strategy the promoting of medications and their control, high exhibition fluid chromatography (HPLC) supplanted various spectroscopic techniques and gas chromatography (GC) in the quantitative and qualitative examination. In the first time of HPLC requisition it was imagined that it might turn into a reciprocal technique for gas chromatography, on the other hand, today HPLC has practically totally swapped gas chromatography in pharmaceutical dissection. The requisition of the fluid versatile stage with the plausibility of conversion of prepared extremity throughout chromatography and all different alterations of portable stage relying on the qualities of substance which are, no doubt, being tried, is an extraordinary focal point in the any time that was spent detachment in examination to different routines. The more terrific decision of stationary stage is the following element which empowers acknowledgement of exceptional detachment. The partition line is joined with particular and touchy indicator frameworks, spectrafluorimeter, diode indicator, electrochemical identifier as other hyphernated frameworks HPLC-MS and HPLC-NMR, are the fundamental components on which is based such wide and successful requisition of the HPLC strategy. The reason high exhibition fluid chromatography dissection of any medications is to affirm the character of a medication and give quantitative effects and additionally to screen the advancement of the help of an infection. Measuring introduced is HPLC chromatogram (information not indicated) got for the plasma of discouraged patients $12 \mathrm{~h}$ preceding oral government of dexamethasone. It might likewise be utilized to further our comprehension of the ordinary and illness handle in the human form trough biomedical and restoratively investigate throughout examination before of the medications enlistment. The investigations of medications and metabolites in organic liquids, especially plasma, serum or pee is a standout amongst the most requesting yet a standout amongst the most well-known employments of high exhibition of fluid chromatography. Blood, plasma or serum holds various endogenous mixes regularly show in focuses much more stupendous than those of analyte. Analyte fixations are regularly level, and on account of pills, the endogenous mixes are now and again structurally fundamentally the same to the medication to be measured. The coupling of pills to the plasma protein additionally might happen which diminishes the measure of free intensify that is measured. To undertake the examinations of pills and metabolites in form liquids the examiner is feature with some issues. The foremost issue is because of the intricate nature of the form liquid, the pills must be separated by an extraction system, for example Strong Stage Extraction (SPE), which conceivably might as well furnish a generally clean remove, and the detachment framework must be equipped for determining the pills of investment from co extractives. AII specified when we are utilizing high exhibition fluid chromatography require great choices of locators, exceptional stationary stage, eluents and satisfactory customize throughout detachment. UV/VIS finder is the most all around adaptable identifier utilized as a part of high exhibition fluid chromatography it is not dependably perfect since it is absence of specificity means high determination of the analyte that may be needed. UV recognition is leaned toward since it offers magnificent linearity and fast quantitative investigations could be performed against a solitary standard of the pill being dead set. Diode cluster and fast checking indicator are functional for crest Id and overseeing crest virtue yet they are sort of less touchy then single wavelength locators. In fluid chromatography a few parts may have unfortunate UV chromophores if UV identification is almost always utilized or be totally held on the fluid chromatography segment. Fluorescence and electrochemical finder are not just extensively more touchy towed proper analytes and yet more particular than UV locators for numerous mixes (at around more than 10 times delicate and specific). In the event that whatsoever conceivable fluorescence finders are delicate, stable, specific and simple to work. It is selectivity demonstrates to itself in the absence of frontal parts watched in plasma remove though electrochemical discovery is practically dependably connected with a major or frontal crest than tails significantly. To date, the most touchy and particular technique has been the reductive electrochemical location and giving the great brings about the examination on a few classes of pills with moderately lower oxidation-diminishment probabilities. Some high exhibition fluid chromatography oxidative electrochemical techniques have been created for the examinations of medications and metabolites in figure liquids. Mass Spectrometer as particular locator with all variety of ionisation and interface (thermo shower, moving sash and so on) or fluid chromatography-tandem mass spectrometry. NMR as specific and particular indicator in high exhibition fluid chromatography today is likewise utilized. The improvement of a non-fluid eluent for particle trade division on silica has furnished a fantastic framework which, when utilized within conjugation with an electrochemical indicator, grants the investigations of a broad extend of particularly fundamental pills and metabolites. New pressing materials, for example polymeric, base deactivated silica's, pyrolysed carbon and the inside surface pressing may as well offer the enhanced steadiness and higher efficiencies for certain classes of the mixes, for example fundamental pills. Microbore segments may as well come to be more acknowledged since they offer not just enhanced affectability and division effectiveness and yet a more level dissolvable utilization and subsequently the diminished ought to discard toxic solvents. Numerous dissections of fundamental pills are still performed by the same technique for the particle trade chromatography on unmodified silica sections with an eluent supported to about ph 9 . Nonpartisan or pitifully acidic medications case in point barbiturates could be chromatographed on a switched stage framework whilst acidic medications for instance paracetamol, cannabis are differentiated either by particle concealment or particle match chromatography on a switched stage pressing material. In micelar fluid chromatography micelar portable stages in switched stage rather than routine hydro natural versatile stage is utilized. In micelar

*Corresponding author: Hideharu Shintani, Chuo University, School of Science 1-13-27, Kasuga Bunkyo 112-0003 Tokyo, Japan, Tel: +81425922336; E-mail shintani@mail.hinocatv.ne.jp

Received June 12, 2013; Accepted June 14, 2013; Published June 17, 2013

Citation: Shintani H (2013) Drug Analysis in Body Fluids. Pharm Anal Acta S2 e001. doi:10.4172/2153-2435.S2-e001

Copyright: (c) 2013 Shintani H. This is an open-access article distributed unde the terms of the Creative Commons Attribution License, which permits unrestricted use, distribution, and reproduction in any medium, provided the original author and source are credited. 
fluid chromatography complex electrostatic hydrophobic and steric face to face times exist between the solute and both stationary and portable stages. These empower the successful partition of specimens of diverse nature. The essential favorable circumstances of the utilization of a micelar result in switched stage fluid chromatography are the dissolvable and the easier expense and danger, the biodegradability of the dissolvable and the simple disintegration of diagnostic specimens that empowers the determination of pills in physiological liquids without the requirement for past detachment of the proteins exhibit in the examples. Utilizing tetrabutylammonium phosphate as a contending base in the examination of sulphonamides and heptanes sulfonate as particle blending reagent. Ion matching reagent is term used to depict improved maintenance as the consequence of the expansion to the portable stage of an imposing particle inverse charge to the atomic particles to be divided. For sub-atomic cations alkyl sulphates or sulfonates are usually used for particle combine investigation in the most cases. I thus portrayed some portable stage, discovery strategy, and issues and settlement for dissecting pills and their metabolite in entangled form liquids by and large. 